\title{
Mulheres submetidas à conização de colo uterino: análise dos resultados citológico e histopatológico
}

\section{Women undergoing conization of cervix: results of cytological and histopathological analysis}

Cláudia Priscilla de Souza Amorim ${ }^{1}$. Ana Beatriz Cavallari Monteiro ${ }^{1}$. Gabriel Ibiapina do Monte Ruben Siqueira ${ }^{1}$. Raquel Autran Coelho ${ }^{1}$.

1. Serviço de Patologia Cervical, Maternidade-Escola Assis Chateaubriand, Universidade Federal do Ceará.

\section{RESUMO}

Objetivo: Relacionar os achados cito-histológicos em pacientes submetidas a procedimento excisional no colo uterino, avaliando as discrepâncias diagnósticas encontradas. Metodologia: Foram avaliadas 52 pacientes submetidas à conização cervical no ambulatório do Setor de Patologia do Trato Genital Inferior da Maternidade-Escola Assis Chateaubriand, atendidas entre janeiro de 2012 a dezembro 2013, retrospectivamente, por revisão de prontuário. Os diagnósticos citopatológicos foram confrontados com os achados colposcópicos e com os laudos histopatológicos para que se observe o nível de concordância. Resultados: A média de idade das pacientes avaliadas foi de 36,5 anos. A média de paridade foi de 3 . Das 21 pacientes com anatomopatológico (AP) de Cirurgia de Alta Frequência (CAF) NIC 3, 3 tinham citologia com Atipias de Células Escamosas de Significado Indeterminado (ASC-US), 3 Lesão intraepitelial de baixo grau (LIEBG) e 15 Lesão intraepitelial de alto grau (LIEAG) em citologia previamente ao procedimento. As 11 pacientes com NIC 2 no AP do CAF, 1 tinha citologia prévia evidenciando ASC-US. Conclusão: Houve boa concordância entre os resultados citológicos e histológicos. Cerca de $20 \%$ das pacientes com NIC 2 ou 3 após tratamento excisional por CAF tinham citologia prévia indicando lesão cervical menor.

Palavras-Chave: Colo do útero. Colposcopia. Neoplasia intraepitelial cervical. Conização.

\section{ABSTRACT}

Objective: This study aims to relate the cytohistological findings in patients undergoing excisional procedure the cervix, evaluating the diagnostic discrepancies found. Methods: We evaluated 52 patients who underwent cervical conization for cervical intraepithelial neoplastic in the clinic of Lower Genital Tract Pathology Sector of Assis Chateaubriand Maternity Hospital, assisted in the period January 2012 and December 2013 retrospectively by chart review. The cytological diagnoses were compared with the colposcopy findings and histopathological results to observe that the level of agreement. Results: The mean age of patients was 36.5 years. The average parity was 3.16 patients with pathology (AP) High Frequency Surgery (CAF) CIN 3, 3 had Atypical Squamous Cells cytology of Undetermined Significance (ASC-US), 3 low-grade squamous intraepithelial lesion (LIEBG) and 15 high-grade squamous intraepithelial lesion (HSIL) in cytology prior to the procedure. The 11 patients with CIN 2 in the CAF AP, 1 had previous cytology showing ASC-US). Conclusion: There was good agreement between the cytological and histological findings. About $20 \%$ of patients with CIN 2 or 3 after excisional treatment CAF had previous cytology indicating lower cervical injury.

Keywords: Cervix uteri. Colposcopy. Cervical intraepithelial neoplasia. Conization.

Autor correspondente: Cláudia Priscilla de Souza Amorim, Universidade Federal do Ceará. E-mail: priamorim6@hotmail.com. Conflito de interesses: Não há qualquer conflito de interesses por parte de qualquer um dos autores.

Recebido em: 16 Abr 2015; Revisado em: 26 Abr 2015; Aceito em: 23 Mai 2015. 


\section{INTRODUÇÃO}

O câncer do colo do útero no Brasil é um dos principais problemas de saúde pública, representando a segunda causa de morte nas mulheres, ficando atrás apenas do câncer de mama e de pele do tipo não melanoma. ${ }^{1}$

A infecção persistente por subtipos oncogênicos do vírus HPV (Papilomavírus Humano), especialmente o HPV-16 e o HPV-18, são responsáveis por cerca de $70 \%$ dos cânceres cervicais. $^{2}$ Inicia-se a partir de uma lesão precursora, curável na quase totalidade dos casos. Trata-se de anormalidades epiteliais conhecidas como neoplasias intraepiteliais cervicais de graus 2 e 3 . As mulheres que desenvolvem infecção persistente por HPV do tipo 16 têm cerca de 5\% de risco de desenvolverem NIC 3 ou lesão mais grave em três anos e $20 \%$ de risco em dez anos. Quando a infecção persistente for por outros tipos de HPV oncogênico, esse risco reduz pela metade. ${ }^{3}$

A realização periódica do exame citopatológico continua sendo a estratégia mais adotada para o rastreamento do câncer do colo do útero. Atingir alta cobertura é o componente mais importante da atenção primária para que se obtenha significativa redução da incidência e da mortalidade por câncer do colo do útero.,

No entanto, diante de sugestiva lesão intraepitelial de alto grau (HSIL), a investigação é realizada a partir de três passos: citologia, colposcopia e biópsia. Para o estudo histopatológico, pode ser feita a cirurgia de alta frequência (CAF), atualmente denominada exérese da zona de transformação (EZT), que consiste em procedimento de baixo custo, podendo ser realizado no ambulatório ou consultório. A remoção completa da NIC, bem como da junção escamo-colunar (JEC), é o principal objetivo dessa técnica. ${ }^{6}$

A peça obtida da conização permite boa avaliação histopatológica. Na peça devem ser avaliados o grau de lesão, as margens cirúrgicas, se comprometidas ou não, e a presença ou não de ocupação glandular, importantes fatores de risco para recidiva de NIC. ${ }^{7}$

Este estudo foi realizado diante de divergências de recomendações para laudos citológicos alterados, além da dificuldade de acesso e seguimento pelas pacientes aos serviços de saúde, especialmente em países em desenvolvimento.

Diante disso, o objetivo deste estudo foi analisar os resultados citológico e histopatológico das pacientes submetidas à CAF, avaliando as discrepâncias diagnósticas encontradas, assim como foram analisados fatores epidemiológicos (tabagismo, uso de $\mathrm{ACH}$, paridade, idade, menarca, coitarca) e sua associação com o desenvolvimento de neoplasia intraepitelial cervical.

\section{METODOLOGIA}

Realizou-se um estudo retrospectivo, realizado na Maternidade-Escola Assis Chateaubriand/UFC de Fortaleza, como parte da monografia.

Este estudo teve como base os resultados dos exames das pacientes submetidas à conização cervical no Ambulatório de Patologia Cervical da Maternidade Escola, no período de janeiro de 2012 a dezembro de 2013, totalizando 52 exames analisados.

Foram revisados 143 prontuários, sendo excluídas do estudo: pacientes gestantes, imunodeprimidas, menores de 18 anos e pacientes submetidas à conização à frio, biopsia excisional por CAF e resultados anatomopatológicos que não descreveram as margens cirúrgicas, se comprometidas ou não.

Foram observados a idade das pacientes, a paridade, a idade da coitarca, o número de parceiros e o tabagismo. Os diagnósticos citopatológicos foram confrontados com os achados colposcópicos e com os laudos histopatológicos para que se observe o nível de concordância.

Os resultados dos exames citopatológicos foram categorizados de acordo com a Nomenclatura Brasileira para Laudos Cervicais em: negativo para neoplasia, células escamosas atípicas de significado indeterminado, possivelmente não neoplásicas (ASC-US); células escamosas atípicas de significado indeterminado, não se pode excluir lesão de alto grau (ASC-H); lesão intraepitelial de baixo grau (LSIL); lesão intraepitelial de alto grau (HSIL); células glandulares atípicas (AGC); adenocarcinoma in situ e carcinomas invasores.

O resultado da biópsia foi classificado em: cervicite crônica, metaplasia escamosa, neoplasia intraepitelial grau 1 (NIC 1), neoplasia intraepitelial grau 2/3 (NIC 2/3), carcinoma escamoso invasivo, adenocarcinoma in situ $\mathrm{e}$ adenocarcinoma invasor.

O programa estatístico utilizado foi Sigmaplot, onde foram usados: análise descritiva para média e DP e foram feitos testes "t" para comparar as variáveis contínuas - idade e teste qui-quadrado para comparar as variáveis qualitativas $(\mathrm{ACH}$ e tabagismo).

\section{RESULTADOS}

O estudo mostrou que dos 52 resultados de exames analisados, 36 tinham laudo citopatológico de lesão de alto grau, 10 de baixo grau, 6 de ASC-US. Quanto ao laudo histopatológico do CAF observou-se 12 NIC 1, 11 NIC 2, 21 NIC 3 e 8 negativos para malignidade. A idade variou de 19 a 61 anos. Das 21 pacientes com AP do CAF NIC 3, 3 tinham citologia ASC-US, 3 LSIL e 15 HSIL. As 11 pacientes com NIC 2 no AP do CAF, 1 tinha citologia prévia evidenciando ASC-US).

\section{DISCUSSÃO}

Diante de resultado citopatológico de ASC-US/LSIL, devese repetir o exame citopatológico após seis meses, pois a 
Tabela 1. Descrição de fatores epidemiológicos das mulheres avaliadas.

\begin{tabular}{ll}
\hline Variáveis & Valores \\
\hline Idade (anos)* & $35,9 \pm 9,6$ \\
Menarca (anos)* & $12,2 \pm 1,1$ \\
Coitarca (anos)* & $17,0 \pm 2,0$ \\
ACH** & \\
Sim & $27(58.8 \%)$ \\
Não & $25(41.2 \%)$ \\
Tabagismo** & \\
Sim & $9(14.7 \%)$ \\
& $43(85.3 \%)$ \\
\hline
\end{tabular}

*Media \pm D.P.; ** N (\%).

Tabela 2. Média de idade das pacientes em cada grupo.

Normal/LSIL HSIL $p$

Idade (anos)* $\quad 33,3 \pm 8,3 \quad 37,6 \pm 10,1 \quad 0,057$

*Media \pm D.P.

Tabela 3. Descrição dos fatores predisponentes em cada grupo.

$\begin{array}{ccc}\text { Normal/LSIL } & \text { HSIL } & p \\ \mathrm{~N}(\%) & \mathrm{N}(\%) & \end{array}$

\section{$\mathrm{ACH}$}

$\begin{array}{llll}\text { Sim } & 9,000(16,4) & 18,000(32,7) & 0,582 \\ \text { Não } & 12,000(21,8) & 16,000(29,1) & \end{array}$

Tabagismo

$\begin{array}{lccc}\text { Sim } & 4(7,3) & 5(9,1) & \\ \text { Não } & 17(30,9) & 29(52,7) & \\ \end{array}$

Tabela 04. Resultados dos exames anatomopatológicos após CAF de 52 mulheres atendidas na Maternidade-Escola, em Fortaleza, no período de Janeiro de 2012 a Dezembro de 2013.

\section{Citologia}

ASC- LSIL HSIL Total US
Anatomopatológico

\begin{tabular}{lcccc}
\hline NORMAL & - & 4 & 4 & 8 \\
NIC 1 & 2 & 3 & 7 & 12 \\
NIC 2 & 1 & - & 10 & 11 \\
NIC 3 & 3 & 3 & 15 & 21 \\
\hline Total & 6 & 10 & 36 & 52 \\
\hline
\end{tabular}

maioria dessas alterações regride espontaneamente, no entanto, caso se mantenha o mesmo resultado ou pior diagnóstico, a paciente deve ser encaminhada para realizar o exame colposcópico com biopsia dirigida. Entretanto, há controvérsia nessa conduta, em um estudo houve prevalência de $10 \%$ de casos de lesões pré-invasivas (HSIL - NIC 2/ 3), mostrando que a conduta conservadora de encaminhar a paciente para colposcopia somente após duas citologias com diagnóstico de ASC-US apenas retarda seu diagnóstico em seis meses. ${ }^{8}$

A colposcopia subsequente ao resultado citopatológico é a melhor forma de identificar as atipias que correspondem a NIC de alto grau. Por esse motivo, a observação das normas e os encaminhamentos corretos são parâmetros importantes para o melhor desempenho do programa de rastreamento e controle do câncer do colo do útero. Além de ser uma alternativa econômica, a conduta preconizada é viável e simples, podendo ser realizada em consultório ginecológico e UABS. $^{9}$

As mulheres com resultados citopatológicos classificados como ASC-H/HSIL, devem ser encaminhadas imediatamente para o exame colposcópico. Após confirmação colposcópica ou histológica, as diretrizes brasileiras recomendam o tratamento excisional das lesões intraepiteliais escamosas de alto grau, por meio de exérese da zona de transformação (EZT) por eletrocirurgia. ${ }^{8}$

Quando a colposcopia é satisfatória, com achado anormal compatível com a citologia, restrito à ectocérvice ou até o primeiro centímetro do canal endocervical, o procedimento deve ser realizado ambulatorialmente, permitindo o tratamento imediato das lesões - prática chamada "Ver e Tratar". O objetivo desta estratégia é facilitar o acesso das mulheres ao tratamento, diminuindo a ansiedade, as possibilidades de perdas no seguimento e os custos da assistência. No caso de colposcopia insatisfatória, ou quando a lesão ultrapassa o primeiro centímetro do canal, o 
tratamento indicado é a conização, realizada preferencialmente por técnica eletrocirúrgica. ${ }^{10}$

Dentre os fatores ambientais associados com infecção do HPV estão a paridade, o uso de contraceptivos orais, o fumo, a coinfecção com o vírus da imunodeficiência humana (HIV) e outras doenças sexualmente transmissíveis (DST) e o hábito alimentar. Estudos epidemiológicos têm demonstrado forte associação entre a multiparidade e as lesões de alto grau e o carcinoma cervical. A International Agency for Research on Cancer (IARC) observou que mulheres com sete ou mais gestações a termo tiveram risco de desenvolver câncer cervical aumentado em quatro vezes, quando comparadas com as nulíparas. ${ }^{11}$

A relação do tempo de uso de contraceptivos orais para a ocorrência de lesão cervical varia de 5 a 9 anos. ${ }^{12-14}$ Parece que mecanismos relacionados ao componente hormonal existente nos anticoncepcionais facilitariam a persistência da infecção viral e a promoção da forma epissomal (não integrada) do DNA-HPV para a integração dentro do genoma do hospedeiro, além de efeitos sobre o próprio epitélio metaplásico. ${ }^{15}$

Entretanto, em outros estudos realizados não houve risco aumentado de desenvolvimento de neoplasia intraepitelial cervical ou câncer cervical em usuárias de contraceptivos orais. Portanto, pelo fato de os estudos serem controversos, não se justifica a interrupção do seu uso em mulheres com diagnóstico de lesões precursoras do câncer cervical. ${ }^{16}$

\section{REFERÊNCIAS}

1. Uchimura NS, Nakano K, Nakano LC, Uchimura TT. Qualidade e desempenho das colpocitopatologias na prevenção de câncer de colo uterino. Rev Assoc Med Bras. 2009;55(5):569-74.

2. ICO Information Centre on HPV and Cancer. Brasil: human papillomavirus and related cancers, fact sheet 2014 [Internet]. Barcelona: ICO HPV Information Centre; 2014 Dec 15; [acesso 2014 jul 20]. Disponível em: http://www.hpvcentre.net/ statistics/reports/BRA FS.pdf

3. Kjaer SK, Frederiksen K, Munk C, Iftner T. Long-term absolute risk of cervical intraepithelial neoplasia grade 3 or worse following human papillomavirus infection: role of persistence. J Natl Cancer Inst. 2010;102(19):1478-88.

4. Anttila A, von Karsa L, Aasmaa A, Fender M, Patnick J, Rebolj $\mathrm{M}$, et al. Cervical cancer screening policies and coverage in Europe. Eur J Cancer. 2009;45(15):2649-58.

5. Arbyn M, Anttila A, Jordan J, Ronco G, Shenck U, Segnan N, et al. European Guidelines for Quality Assurance in Cervical Cancer Screening. Second edition - summary document. Ann Oncol. 2010;21(3):448-58.

6. Russomano F. Cirurgia de alta frequência [Internet]. Rio de Janeiro: Associação Brasileira de PTGI e Colposcopia; c2013 [acesso 2009 out 25]. Disponível em: http://www.colposcopia.org.br/espaco_mulher/dicas/cirurgia-de-alt

7. Livasy CA, Maygarden SJ, Rajaratnam CT, Novotny DB. Predictors of recurrent dysplasia after a cervical loop electrocautery excision procedure for CIN-3: a study of margin,
Evidências epidemiológicas demonstraram a associação entre o hábito de fumar e o risco de desenvolvimento de neoplasias intraepiteliais cervicais. O risco estimado nas fumantes é em torno de duas vezes maior do que nas não fumantes e está intimamente relacionado ao uso prolongado e ao número de cigarro. O tabaco diminui a concentração de células de Langerhans no colo uterino, favorecendo a persistência viral, que é importante fator contribuinte para o aparecimento de lesões cervicais pré-malignas e malignas. ${ }^{17}$

Neste estudo não se evidenciou diferença entre os grupos quanto ao uso de anticoncepcional hormonal (ACH) e o tabagismo no aumento do desenvolvimento de neoplasias intraepiteliais cervicais, podendo estar relacionado ao número pequeno de pacientes avaliadas.

\section{CONCLUSÃO}

Um percentual significativo das pacientes com estudo histopatológico revelando NIC 2 ou 3 após tratamento excisional por CAF tinha citologia prévia indicando lesão cervical menor. Os dados são concordantes com a literatura vigente, que sugere referência a colposcopia para pacientes com LIEBG de colo, ao invés de seguimento citológico semestral.

Não se evidenciou diferença entre os grupos quanto à idade $\mathrm{e}$ ao uso de anticoncepcional hormonal $(\mathrm{ACH})$ e ao tabagismo no aumento do desenvolvimento de neoplasias intraepiteliais cervicais.

endocervical gland, and quadrant involvement. Mod Pathol. 1999;12(3):233-8.

8. Instituto Nacional de Câncer (Brasil). Nomenclatura brasileira para laudos cervicais e condutas preconizadas: recomendações para profissionais de saúde. 2. ed. Rio de Janeiro, INCA; 2006.

9. Pedrosa ML. Perfil epidemiológico de mulheres portadoras de atipias escamosas de significado indeterminado atendidas pelo programa de controle do câncer de colo uterino no município do Rio de Janeiro. Rio de Janeiro: Fundação Oswaldo Cruz; 2003.

10. Instituto Nacional de Câncer (Brasil). Plano de ação para redução da incidência e mortalidade por câncer do colo do útero: sumário executivo. Rio de Janeiro: INCA; 2010.

11. Muñoz N, Franceschi S, Bosetti C, Moreno V, Herrero R, Smith $\mathrm{JS}$, et al. Role of parity and human papillomavirus in cervical cancer: the IARC multicentric case-control study. Lancet. 2002;359(9312):1093-1101.

12. Deacon JM, Evans CD, Yule R, Desai M, Binns W, Taylor C, et al. Sexual behaviour and smoking as determinants of cervical HPV infection and of CIN3 among those infected: a case-control study nested within the Manchester cohort. $\mathrm{Br} \mathrm{J}$ Cancer. 2000;83(11):1565-72.

13. Kruger-Kjaer S, van den Brule AJ, Svare EI, Engholm G, Sherman ME, Poll PA, et al. Different risk factor patterns for highgrade and low-grade intraepithelial lesions on the cervix among $\mathrm{HPV}$-positive and HPV-negative young women. Int $\mathrm{J}$ Cancer. 1998;76(5):613-9. 
14. Smith JS, Green J, Berrington de Gonzales A, Appleby P, Peto $\mathrm{J}$, Plummer $\mathrm{M}$, et al. Cervical cancer and use of hormonal contraceptives: a systematic review. Lancet. 2003;361(9364):115967.

15. Swan SH, Petitti DP. A review of problems of bias and confounding in epidemiologic studies of cervical neoplasia and oral contraceptive use. Am J Epidemiol. 1982;115(1):10-8.

16. Schiffman MH, Bauer HM, Hoover RN, Glass AG, Cadell DM, Rush BB, et al. Epidemiologic evidence showing that human papillomavirus infection causes most cervical intraepithelial neoplasia. J Natl Cancer Inst. 1993;85(12):958-64.

17 International Collaboration of Epidemiological Studies of Cervical Cancer; Appleby P, Beral V, Berrington de González A, Colin D, Franceschi S, et al. Carcinoma of the cervix and tobacco smoking: collaborative reanalysis of individual data on 13,541 women with carcinoma of the cervix and 23,017 women without carcinoma of the cervix from 23 epidemiological studies. Int $\mathrm{J}$ Cancer 2006;118(6):1481-95.

\section{Como citar:}

Amorim CP, Monteiro AB, Siqueira GI, Coelho RA. Mulheres submetidas à conização de colo uterino: análise dos resultados citológico e histopatológico. Rev Med UFC. 2015 jan-jun;55(1):13-17. 